\title{
The effect of daily baked bean (Phaseolus vulgaris) consumption on the plasma lipid levels of young, normo-cholesterolaemic men
}

\author{
BY SUSAN M. SHUTLER ${ }^{1,3, *}$, GEMMA M. BIRCHER 2 , \\ JACKI A. TREDGER ${ }^{2}$, LINDA M. MORGAN ${ }^{2}$, ANN F. WALKER ${ }^{3}$ \\ AND A. G. L.OW' \\ ${ }^{1}$ Pig Department, AFRC Institute for Grassland and Animal Production, Shinfield, \\ Reading, RG2 $9 A Q$ Berks. \\ ${ }^{2}$ Department of Biochemistry, University of Surrey, Guildford, Surrey GU2 $5 X H$ \\ ${ }^{3}$ Department of Food Science and Technology, University of Reading, Whiteknights, \\ Reading, RG6 2AP Berks.
}

\section{(Received 7 April 1988 - Accepted 9 November 1988)}

\begin{abstract}
1. Thirteen normo-cholesterolaemic male students consumed one $450 \mathrm{~g}$ can of baked beans (Phaseolus vulgaris) in tomato sauce, daily, for $14 \mathrm{~d}$ as part of their normal diet. After a $14 \mathrm{~d}$ washout period, eleven of the students went on to consume one $440 \mathrm{~g}$ can of spaghetti in tomato sauce, daily, for $14 \mathrm{~d}$.

2. Fasting blood samples were taken frequently for measurement of plasma cholesterol, high-density lipoprotein (HDL)-cholesterol, triacylglycerols, glucose, insulin and C-peptide. Diet diaries ( $3 \mathrm{~d}$ ) were completed by the subjects during each period.

3. Consumption of beans and spaghetti led to a significant reduction in the amount of fat eaten daily $(P<0.05)$. Bean consumption also resulted in significant increases in protein, fibre and sugar intakes $(P<0.02, P<0.001$ and $P<0.05$ respectively).

4. During the bean-eating period the mean total plasma cholesterol level of the students fell significantly from 5.1 to $4.5 \mathrm{mmol} / 1(P<0.02)$. No reduction in plasma cholesterol occurred during the spaghetti-eating period.

5. HDL-cholesterol levels fell significantly during both periods $(P<0-001)$, but HDL: total cholesterol ratio was significantly reduced only during the spaghetti-eating period $(P<0.001)$. Neither beans nor spaghetti affected triacylglycerol, insulin or C-peptide levels.

6. The benefits of a legume-rich diet are discussed.
\end{abstract}

In recent years, much interest has been shown in the beneficial effects of diets based on legumes for diabetic subjects (e.g. Simpson et al. 1981). Increasing evidence suggests that legume-based diets may also be of benefit to hyperlipidaemic patients. A number of studies have shown substantial reductions in plasma cholesterol levels of hyperlipidaemic human subjects consuming a variety of dried (boiled or canned) legumes on a regular basis (Bingwen et al. 1981; Jenkins \& Jepson, 1982; Jenkins et al. 1983; Anderson et al. 1984).

As a general rule, mature legume seeds are not popular foods in Britain. However, the popularity of the canned product, baked beans (Phaseolus vulgaris L.) in tomato sauce, is exceptional. Household expenditure values for the United Kingdom show that the consumption of canned beans is higher than that of any other vegetable, except potatoes (Ministry of Agriculture, Fisheries and Food, 1985). This means that, as an accepted food item, baked beans have unique potential for use in therapeutic or prophylactic diets for coronary heart disease.

The effect of a diet based on legumes, other than soya beans, on the plasma lipid levels of normo-lipidaemic subjects has been studied in only two trials. In the first (Mathur et al. 1968), high intakes of chick peas (Cicer arietinum L.) reduced plasma cholesterol in twenty men consuming a butter-rich diet. In the second (Simpson et al. 1981), twenty-seven

\footnotetext{
* Present name and address: Susan M. Kingman, Dunn Clinical Nutrition Centre, 100 Tennis Court Road, Cambridge CB2 1QL.
} 
diabetic subjects showed a significant reduction in mean plasma cholesterol level after 6 weeks on a diet rich in leguminous seeds of mixed species.

The aim of the present experiment was to investigate the effect of daily baked-bean consumption on the plasma lipid levels of normal, healthy, human volunteers.

Attention was paid to changes in the nutrient profile of the diet resulting from the inclusion of legumes, and to relations between these and plasma lipid levels. Fasting levels of plasma glucose, insulin and C-peptide were also measured.

\section{MATERIALS AND METHODS}

Subjects

Thirteen male undergraduate students (age range 18-26 years) were recruited from the University of Surrey. All were non-smokers and free from overt disease, with plasma cholesterol levels ranging between 3.45 and $6.39 \mathrm{mmol} / 1$. Subjects were given full details of the study protocol and gave verbal consent to participate. University ethical guidelines were adhered to throughout the trial. For 4 weeks immediately preceding the trial, all subjects were asked to refrain from eating baked beans.

\section{Diets}

The products used were baked beans in tomato sauce (Heinz; $450 \mathrm{~g}$ cans) and spaghetti in tomato sauce (Heinz; $440 \mathrm{~g}$ cans). Canned spaghetti was chosen as the control product because it is similar in composition to baked beans (in terms of starch, sugar, fat, sodium, cholesterol and energy contents) and is eaten in similar circumstances.

Subjects were encouraged to maintain their usual diet (quantity and style) as far as possible throughout the experiment, to minimize additional sources of variation.

\section{Study protocol}

Baseline fasting plasma lipid levels for each subject were taken to be the mean values from two blood samples taken $5 \mathrm{~d}$ apart before the start of the experiment. Baseline dietary information was collected as described later (p. 259). The study consisted of three consecutive $14 \mathrm{~d}$ periods: test, washout and control.

\section{Test period}

Each subject was asked to consume one $450 \mathrm{~g}$ can of baked beans in tomato sauce, daily, as part of his normal diet. Blood samples $(10 \mathrm{ml})$ were taken twice weekly, after an overnight fast, the last sample being taken on the day after the last tin of beans had been consumed.

\section{Washout period}

Subjects returned to their normal diet, but were asked to refrain from eating baked beans, or other legumes, for the rest of the study. Fasting blood samples were taken on the 9th and 14 th days of this period.

\section{Control period}

Each subject was asked to consume one $440 \mathrm{~g}$ can of spaghetti in tomato sauce, daily, as part of his normal diet. Fasting blood samples were taken as for the test period.

A final blood sample was taken $17 \mathrm{~d}$ after the completion of the spaghetti-eating period.

\section{Biochemical methods}

Blood samples were immediately centrifuged after collection, in lithium heparinized tubes (or fluoridated tubes for glucose determination). The plasma samples were stored frozen before analysis. 
All lipid analyses were carried out using a fully automated clinical analyser (ENCORE Clinical Chemistry System, Baker Instruments) and enzymic kits. The following variables were measured.

Total plasma cholesterol. ENCORE cholesterol reagent set no. 27-012-700-000 (Baker Instruments) was used. The method was that of Allain et al (1974) combined with the phenol 4-amino antipyrine system of Trinder (1969). The inter- and intra-assay coefficients of variation were both $\leqslant 2 \cdot 5 \%$.

High-density lipoprotein (HDL)-cholesterol. ENCORE HDL-cholesterol reagent set no. 27-017-365-009 (Baker Instruments) was used. Low- and very low-density lipoproteins were precipitated with dextran sulphate (molecular weight 50000) and magnesium chloride, before estimation of cholesterol by the method described previously. This precipitation procedure has been shown to have good specificity for HDL-separation (Warnick et al. $1982,1983)$, and the total HDL values obtained are not significantly altered by short-term storage at $-20^{\circ}$ (Matthew \& Duggan, 1988). Inter- and intra-assay coefficients of variation were both $\leqslant 2.5 \%$.

Triacylglycerols. ENCORE triglycerides/INT reagent set no. 27-013-900-000 (Baker Instruments) was used. The method was an adaptation of the triacylglycerol lipase (EC 3.1.1.3) hydrolysis-glycerol kinase (EC 2.7.1.3) method of Megraw et al. (1979). Interand intra-assay coefficients of variation were 3.9 and $<2.0 \%$ respectively.

Plasma glucose. Glucose was estimated using a Roche Cobas Bio clinical analyser in conjunction with a hexokinase ( $E C$ 2.7.1.1) glucose-6-phosphate dehydrogenase (EC 1.1.1.49) enzymic kit (Uni-kit III Roche Glucose HK). Inter-assay coefficient of variation was $3 \cdot 3 \%$.

Plasma insulin. Plasma immunoreactive insulin was estimated by double-antibody techniques using antisera from Guildhay Antisera Ltd (Guildford). The sensitivity of the method was $2.5 \mathrm{mU} / 1$, and the inter-assay coefficient of variation was $7.4 \%$.

Plasma C-peptide. Plasma C-peptide was measured by the method of Hampton (1983) using antisera supplied by Guildhay Antisera Ltd (Guildford). The sensitivity of the method was $0.1 \mu \mathrm{g} / \mathrm{l}$, and the inter-assay coefficient of variation $9.0 \%$.

Dietary analysis. Dietary information was obtained for the pre-trial, bean and spaghetti periods, using a $3 \mathrm{~d}$ food diary. Using a printed diary record sheet, each subject recorded his own food and beverage intake on two weekdays and one weekend day within the period. Instructions and illustrative portion sizes were given with each diary, and some subjects retained the labels from manufactured foods, or included recipes.

The diaries were analysed using the University of Surrey 'Compeat' program which is based on the food tables of McCance and Widdowson (Paul \& Southgate, 1978). A standard daily assumed amount of salt $(1 \mathrm{~g})$ was added if the subject had used salt in cooking or at the table.

\section{Statistical methods}

Comparison of the results obtained was carried out using Student's $t$ test for paired values. Relations between the values were established using Pearson's correlation coefficient, $r$.

\section{RESULTS}

Fig. 1 shows the changes in mean plasma cholesterol level which occurred during the study. After only $3 \mathrm{~d}$ on the beans, the mean plasma cholesterol level had fallen by 0.42 (SE 0.12 ) mmol/1 $(P<0.02)$, and it continued to be lower than the baseline level throughout the bean-eating period. The mean reduction observed after $14 \mathrm{~d}$ was 0.59 (SE 0.19 ) $\mathrm{mmol} / \mathrm{l}$ $(11.7 \%, P<0.02$, Table 1$)$. The maximum individual reduction in plasma cholesterol was 


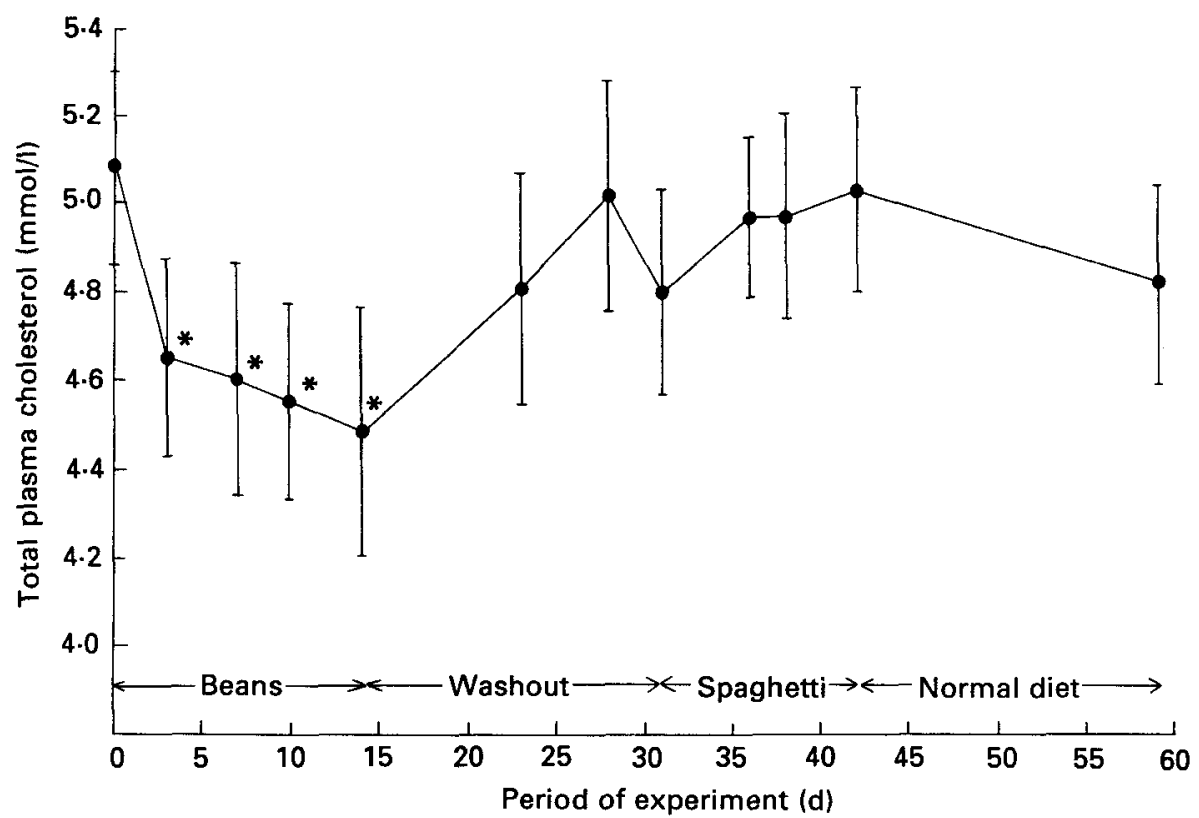

Fig. 1. Change in mean plasma cholesterol level of healthy young men during daily consumption of baked beans (Phaseolus vulgaris) or spaghetti. For details of experimental periods, see p. 258. * Mean values were significantly different from baseline values: $P<0.02$.

$1.79 \mathrm{mmol} / 1(38 \%)$. At the end of the bean-eating period, two subjects had cholesterol levels which were greater than their baseline levels, but in neither case was this consistent for the entire bean-eating period.

After the washout period the mean total cholesterol level had increased by $7.4 \%$ $(P<0.02)$ compared with the bean-eating period, to a value which was no longer significantly different from baseline. During the spaghetti-eating period and the post-trial period, no further significant changes in plasma cholesterol level were observed (Table 1).

Individual baseline HDL-cholesterol levels were within the accepted normal range and varied between 0.93 and $1.61 \mathrm{mmol} / 1$ (mean $1.35 \mathrm{mmol} / 1$, Table 1). During the bean-eating period, HDL-cholesterol levels fell in all subjects to a mean of $1 \cdot 15 \mathrm{mmol} / 1(14.9 \%$, $P<0.001)$. In contrast to total cholesterol, there was no increase in the HDL-cholesterol level during the washout or spaghetti-eating periods. After $17 \mathrm{~d}$ on their normal diets, the subjects continued to show a significant reduction in mean HDL-cholesterol compared with the original baseline value $(10.0 \%, P<0.02$, Table 1$)$.

Calculation of HDL : total cholesterol ratio from these values showed that, whereas the consumption of baked beans reduced total plasma cholesterol levels, it did not alter significantly the original HDL:total cholesterol ratio of $0 \cdot 27$. In contrast, as total cholesterol levels rose during the washout and spaghetti-eating periods, the ratio was reduced, reaching a minimum mean value of 0.21 at the end of the spaghetti-eating period $(P<0.001)$. However, $17 \mathrm{~d}$ later the ratio had returned to a mean value of $0 \cdot 26$, which was not significantly different from baseline. Mean values with their standard errors for HDL: total cholesterol ratios are given in Table 1.

There was no significant effect on the mean plasma triacylglycerol level during any part of the study (Table 1). However, the response of subject PP is worthy of note. This subject had a baseline triacylglycerol level of $2.07 \mathrm{mmol} / 1$, which is just above the normal range 
Baked beans and plasma lipid levels

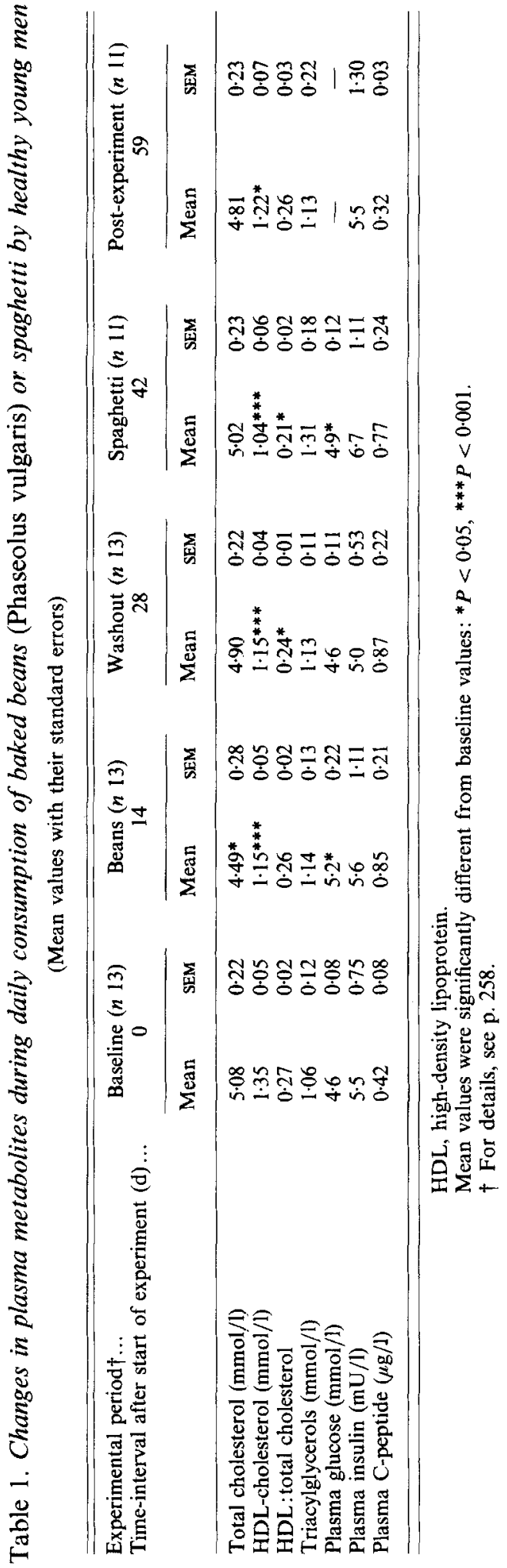


Table 2. Mean daily nutrient intakes of healthy, young men eating their normal diet or a diet in which baked beans (Phaseolus vulgaris) or spaghetti were included on a daily basis

(Mean values and standard deviations)

\begin{tabular}{|c|c|c|c|c|c|c|}
\hline \multirow{2}{*}{$\begin{array}{l}\text { Experimental period } \neq \ldots \\
\text { Intake }\end{array}$} & \multicolumn{2}{|c|}{ Baseline ( $n$ 13) } & \multicolumn{2}{|c|}{ Beans ( $n$ 13) } & \multicolumn{2}{|c|}{ Spaghetti control ( $n$ 11) } \\
\hline & Mean & SD & Mean & SD & Mean & SD \\
\hline Energy $(\mathrm{kJ})$ & 2463 & 290 & 2433 & 325 & 2300 & 321 \\
\hline Protein (g) & 86 & 17 & $98 *+$ & 17 & 78 & 15 \\
\hline Fat $(g)$ & 100 & 17 & $84^{*}$ & 19 & $84^{*}$ & 18 \\
\hline Carbohydrate $(\mathrm{g})$ & 275 & 34 & 302 & 47 & 282 & 43 \\
\hline Starch (g) & 141 & 25 & 158 & 31 & 146 & 40 \\
\hline Sugars (g) & 111 & 30 & $131 * \dagger$ & 35 & 108 & 38 \\
\hline Dietary fibre (g) & 25 & 8 & $60^{*}+$ & 9 & 24 & 7 \\
\hline Alcohol (g) & 26 & 24 & 18 & 16 & 24 & 20 \\
\hline $\mathrm{P}: \mathrm{S}$ & 0.4 & $0 \cdot 16$ & 0.42 & 0.17 & 0.42 & $0 \cdot 24$ \\
\hline Cholesterol (mg) & 291 & 151 & 208 & 147 & 174 & 105 \\
\hline Salt (g) & $9 \cdot 6$ & 1.9 & $13 \cdot 2^{*}$ & $2 \cdot 3$ & $12 \cdot 7^{*}$ & $2 \cdot 1$ \\
\hline
\end{tabular}

$P: S$, polyunsaturated: saturated fatty acid ratio.

Mean values were significantly different from baseline values: $* P<0.05$.

Mean values were significantly different from spaghetti control values: $\dagger P<0.05$.

$\ddagger$ For details, see p. 258.

(0.77-1.98 mmol/1) and consistent with Type IV hyperlipidaemia. During the bean-eating period, this level fell by $56 \%$ to $0.90 \mathrm{mmol} / 1$, but subsequently it rose sharply, reaching a level of $2.83 \mathrm{mmol} / 1$ after the spaghetti-eating period. Following $17 \mathrm{~d}$ on his normal diet the subject's triacylglycerol level was increased further to $3.15 \mathrm{mmol} / 1$.

\section{Plasma glucose, insulin and C-peptide}

Mean (with SEM) plasma levels of glucose, insulin and C-peptide are shown in Table 1 . The individual levels of these metabolites were generally within the normal fasting ranges. A few notable exceptions suggested that some subjects had not adhered strictly to the overnight fast. These samples were excluded when calculating the mean values.

A small rise in plasma glucose was noted during both bean- and spaghetti-eating periods $(P<0.05)$, compared with baseline values, which could not be explained by lack of adherence to the fast.

Lack of adherence to the fast would be expected to have negligible effect on plasma cholesterol and HDL-cholesterol levels (Mayer et al. 1978; Henderson et al. 1980), but may have affected triacylglycerol levels slightly.

\section{Dietary changes}

The changes in nutrient intake which resulted from the inclusion of baked beans and spaghetti in the diet on a daily basis are summarized in Table 2.

A significant reduction $(16 \%, P<0.05)$ in the amount of fat consumed was observed during both periods, and in each case this was accompanied by an increase in the percentage of energy derived from carbohydrate $(P<0.05)$. However, individual changes in fat and carbohydrate consumption were found to be unrelated to changes in plasma cholesterol level. The only other change common to both bean- and spaghetti-eating periods was a highly significant increase in sodium consumption $(37.5 \%, P<0.001)$.

Dietary changes peculiar to the bean-eating period were increases in daily protein 
consumption $(14 \%, P<0.05)$, dietary fibre intake $(134 \% ; P<0.001)$ and sugar intake $(18 \%, P<0.05)$ over baseline intakes. Consumption of these three nutrients during the bean-eating period was also significantly greater than during the spaghetti-eating period (protein $P<0.05$, fibre $P<0.001$, sugar $P<0.05$ ). On an individual level the changes in intake of these nutrients were not found to be related to the reduction in plasma cholesterol.

Compliance with the diets, assessed by one of us (G.M.B.) by unannounced visits to the homes of the subjects, was good for both periods, but two subjects (SD and MC) declined to continue after the bean-eating period. There were no complaints of gastrointestinal discomfort or abnormal degrees of flatulence.

\section{DISCUSSION}

In the present study, the inclusion of $450 \mathrm{~g}$ baked beans in the daily diet of normocholesterolaemic men resulted in a significant reduction in the mean plasma cholesterol level within 1 week. In contrast, no reduction in plasma cholesterol was observed when $440 \mathrm{~g}$ spaghetti in tomato sauce was included in the daily diet of the same men, for a further 2 weeks. During both the dietary-intervention periods, the subjects ate less fat, proportionately more carbohydrate and more $\mathrm{Na}$ than they did on their usual diets. However, the observed changes in plasma cholesterol could not be explained entirely in terms of fat displacement from the diet.

The three dietary components which emerged as possible influences on plasma cholesterol level were dietary fibre, sugar and protein. Previous workers have suggested that the dietary fibre of legumes is the component most likely to affect plasma lipids. Although the change in fibre intake was not found to be closely related to the change in plasma cholesterol, this finding does not preclude an effect of fibre on plasma cholesterol levels. It may simply indicate that the response to a given level of dietary fibre is highly variable between individuals, as might be the case if gut flora were involved. In the study of Anderson et al. (1984), in which haricot beans lowered plasma cholesterol levels in ten hyperlipidaemic men, the effect was attributed to the inhibition of hepatic cholesterol synthesis by volatile fatty acids produced fermentatively from the soluble fibre by gut flora.

The diet diaries indicated that some displacement of animal protein by vegetable protein may have occurred during the bean-eating period. Vegetable proteins have been shown to have hypocholesterolaemic properties in animal studies (for review, see Terpstra et al. 1983), although their influence on human plasma lipids is not well defined (Sacks et al. 1983). Several mechanisms have been proposed to explain the observed cholesterolreducing properties of vegetable proteins. These generally implicate the differences in amino acid composition known to exist between animal and vegetable proteins, and suggest ways in which altered amino acid ratios may affect hepatic cholesterol metabolism, either directly or via the endocrine system. In his review of the hypocholesterolaemic effect of soya-bean proteins, Gibney (1982) suggested a scheme involving insulin and glucagon, two hormones which are known to be correlated positively and negatively respectively, with the activity of rat hepatic hydroxymethylglutaryl-CoA reducase $(E C$ 1.1.1.88; the rate-limiting enzyme in cholesterol synthesis). Gibney (1982) proposed that the release of arginine following soya-bean protein digestion leads to an enhanced stimulation of insulin release. This in turn activates the reductase enzyme, leading to an increase in the turnover of verylow-density lipoproteins. We did not detect any sustained increase in insulin or C-peptide production. This contrasts with the usual finding that diets high in carbohydrate and fibre generally lower fasting insulin levels, and reduce circulating glucose and insulin concentrations following oral glucose administration in normal subjects (Anderson, 1982). 
The effect is, however, dependent on the type of dietary fibre consumed (Jenkins et al. 1978).

It is possible that the observed increase in sugar consumption may have contributed to some extent to the fall in plasma cholesterol levels during the bean-eating period. Nestel et al. (1979) observed markedly decreased total plasma cholesterol levels after normal subjects received a high-sucrose diet, while Reiser et al. (1979) found the opposite in a group of subjects who tended to have elevated plasma lipoprotein levels. However, it is important to note that the increase in sugar consumption in the present study was much smaller than in the two studies referred to previously.

Whilst the results of the present study have suggested dietary fibre and protein as possible influencing factors in the cholesterol-lowering effect of baked beans, other, minor components are also thought to contribute to the effect. These have recently been reviewed by Shutler et al. $(1987 a, b)$.

HDL-cholesterol fell during the bean- and spaghetti-eating periods, suggesting that the reduction was associated with the dietary changes common to both, i.e. decreased fat and cholesterol intakes. Indeed, several studies have suggested that the level of fat consumption is a major determinant of the HDL-cholesterol level (Knuiman et al. 1983; Mensink \& Katan, 1987). High-fat diets tend to increase HDL-cholesterol levels, whereas low-fat diets have the opposite effect. Sucrose has also been associated with low HDL-cholesterol levels in epidemiological studies (Williams et al. 1985), and Yudkin et al. (1986) found that doubling the sucrose intake of young men led to a $16 \%$ decrease in their mean HDLcholesterol level. However, whilst the change in sugar intake experienced during the beaneating period in the present experiment was significant, it was much smaller than any of the dietary manipulations which have elicited such a response, and it is unlikely that this was the primary cause of the observed effect.

We suggest, therefore, that a diet rich in legumes may be of great benefit to health, not only for diabetic and hyperlipidaemic subjects, but also for the general population.

S. M.S. is in receipt of a MAFF postgraduate award. The authors are grateful to Heinz Ltd for their generous supply of baked beans and spaghetti. Thanks go to H. A. Smith for expert technical assistance.

\section{REFERENCES}

Allain, C. C., Poon, L. S., Chan, C. S. G., Richmond, W. \& Fu, P. C. (1974). Enzymatic determination of total serum cholesterol. Clinical Chemistry 20, 470-475.

Anderson, J. W. (1982). Dietary fibre and diabetes. In Dietary Fibre in Health and Disease, pp. 151-167 [G. V. Vahouny and D. Kritchevsky, editors]. London: Plenum Press

Anderson, J. W., Story, L., Sieling, B., Chen, W.-J. L., Petro, M. S. \& Story, J. (1984). Hypocholesterolaemic effects of oat-bran or bean intake for hypercholesterolaemic men. American Journal of Clinical Nutrition $\mathbf{4 0}$, $1146-1155$.

Bingwen, L., Zhaofeng, W., Whanzhen, L. \& Rongjue, Z. (1981). Effects of bean meal on serum cholesterol and triglycerides. Chinese Medical Journal 94, 455-458.

Gibney, M. J. (1982). Hypocholesterolaemic effect of soya-bean proteins. Proceedings of the Nutrition Society 41, 19-26.

Hampton, S. M. (1983). The C-peptide of proinsulin, its diagnostic use and a possible physiological role, $\mathrm{PhD}$ Thesis, University of Surrey.

Henderson, L. O., Saritelli, A. L., Lagarde, E., Herbert, P. N. \& Shulman, R. S. (1980). Minimal within-day variation of high-density lipoprotein cholesterol and apolipoprotein A-1 levels in normal subjects. Journal of Lipid Research 21, 935-955.

Jenkins, D. J. A. \& Jepson, E. M. (1982). Leguminous seeds and their constituents in the treatment of hyperlipidaemia and diabetes. In Lipoproteins and Coronary Atherosclerosis, pp. 247-256 [G. Noseda, C. Fragiacomo, R. Fumagalli and R. Paoletti, editors]. Amsterdam: Elsevier Biomedical Press.

Jenkins, D. J. A., Wollever, P. M. S., Leeds, A. R., Gassull, M. A., Haisman, P., Dilawari, J. B., Goff, D. V., Metz, G. L. \& Alberti, K. G. M. M. (1978). Dietary fibres, fibre analogues, and glucose tolerance: importance of viscosity. British Medical Journal i, 1392-1394. 
Jenkins, D. J. A., Wong, G. S., Patten, R., Bird, J., Hall, M., Buckley, B. C., McGuire, V., Reichert, R. \& Little, J. A. (1983). Leguminous seeds in the dietary management of hyperlipidaemia. American Journal of Clinical Nutrition 38, 567-573.

Knuiman, J. T., Westenbrink, S., Van der Heijden, L., West, C. E., Burema, J., De Boer, J., Hautvast, J. G. A. J., Rasanen, L., Virkkunen, L., Viikari, J., Lokko, P., Pobee, J. O. M., Ferro-Luzzi, A., Ferrini, A. M., Scaccini, C., Serre, S., Villavieja, G. M. \& Bulatao-Jayme, J. (1983). Determinants of total and high-density lipoprotein cholesterol in boys from Finland, The Netherlands, Italy, the Philippines and Ghana with special reference to diet. Human Nutrition: Clinical Nutrition 37C, 237-254.

Mathur, K. S., Khan, M. A. \& Sharma, R. D. (1968). Hypocholesterolaemic effect of Bengal gram. British Medical Journal i, 30-31.

Matthew, A. \& Duggan, P. F. (1988). Stability of high-density lipoprotein subfractions in stored plasma. Clinical Chemistry 34, 425-426.

Mayer, K. H., Stamler, J., Dyer, A. R., Stamler, R. \& Berkson, D. M. (1978). Epidemiologic findings on the relationship of time of day and time since last meal to five clinical variables: serum cholesterol, haematocrit, systolic and diastolic blood pressure, and heart rate. Preventative Medicine 7, 22-27.

Megraw, R. E., Dunn, D. E. \& Biggs, H. E. (1979). Manual and continuous-flow colorimetry of triacylglycerols by a fully enzymic method. Clinical Chemistry 25, 273-278.

Mensink, R. P. \& Katan, M. B. (1987). Effect of monounsaturated fatty acids versus complex carbohydrates on high density lipoproteins in healthy men and women. Lancet i, 122-124.

Ministry of Agriculture, Fisheries and Food (1985). Household food consumption and expenditure. Annual Report of the National Food Survey Committee, p. 61. London: H.M. Stationery Office.

Nestel, P. J., Reardon, M. \& Fidge, N. H. (1979). Sucrose-induced changes in VLDL- and LDL-B apoprotein removal rates. Metabolism 28, 531-535.

Paul, A. A. \& Southgate, D. A. T. (1978). McCance and Widdowson's The Composition of Foods. London: H. M. Stationery Office.

Reiser, S., Hallfrisch, J., Michaelis, O. E., Lazar, F. L., Martin, R. E. \& Prather, E. S. (1979). Isocaloric exchange of dietary starch and sucrose in humans. 1. Effects on levels of fasting blood lipids. American Journal of Clinical Nutrition 32, 1659-1669.

Sacks, F. M., Breslow, J. L., Wood, P. G. \& Kass, E. H. (1983). Lack of an effect of dietary protein (casein) and soy protein on plasma cholesterol of strict vegetarians. An experiment and critical review. Journal of Lipid Research 24, 1012-1020.

Shutler, S. M., Walker, A. F. \& Low, A. G. (1987a). The cholesterol-lowering effects of legumes. 1. Effects of the major nutrients. Human Nutrition: Food Sciences and Nutrition 41F, 71-86.

Shutler, S. M., Walker, A. F. \& Low, A. G. (1987 b). The cholesterol-lowering effects of legumes. 2. Effects of fibre, sterols, saponins and isoflavones. Human Nutrition: Food Sciences and Nutrtion 41F, 87-102.

Simpson, H. C. R., Simpson, R. W., Lousley, S., Carter, R. D., Geekie, M., Hockaday, T. D. R. \& Mann, J. I. (1981). A high carbohydrate leguminous seed fibre diet improves all aspects of diabetic control. Lancet $\mathbf{i}$, $1-5$.

Terpstra, A. H. M., Hermus, R. J. J. \& West, C. E. (1983). The role of dietary protein in cholesterol metabolism. World Review of Nutrition and Dietetics 42, 1-55.

Trinder, P. (1969). Determination of glucose in blood using glucose oxidase with an alternative oxygen acceptor Annals of Clinical Biochemistry 6, 24-27.

Warnick, G. R., Benderson, J. \& Albers, J. J. (1982). Dextran-sulfate-Mg' ${ }^{2+}$ precipitation procedure for quantitation of high-density lipoprotein cholesterol. Clinical Chemistry 28, 1379-1388.

Warnick, G. R., Benderson, J. \& Albers, J. J. (1983). Dextran-sulfate- $\mathbf{M g}^{2+}$ precipitation procedure for quantitation of high-density-lipoprotein cholesterol. Selected Methods for Clinical Chemistry 10, 91-99.

Williams, P. T., Krauss, R. M., Wood, P. D., Albers, J. J., Dreon, D. \& Ellsworth, N. (1985). Associations of diet and alcohol intake with high density lipoprotein subclasses. Metabolism 34, 524-530.

Yudkin, J., Eisa, O., Kang, S. S., Meraji, S. \& Bruckdorfer, K. R. (1986). Dietary sucrose affects plasma HDL concentrations in young men. Annals of Nutrition and Metabolism 30, 261-266. 